\title{
A versatile and low-cost 3D acquisition and processing pipeline for collecting mass of archaeological findings on the field
}

\author{
E. Gattet ${ }^{a}$, J. Devogelaere ${ }^{b}$, R. Raffin, L. Bergerot ${ }^{a}$, M. Daniel ${ }^{c}$, Ph. Jockey ${ }^{b}$, L. De Luca ${ }^{a}$ \\ a UMR CNRS/MCC MAP 3495 - (eloi.gattet, laurent.bergerot, livio.deluca)@map.cnrs.fr \\ b Aix-Marseille University, CNRS, UMR 77299 CCJ, LabexMed (devogelaere@mmsh.univ-aix.fr, philippe.jockey@univ-amu.fr) \\ c Aix-Marseille University, CNRS LSIS UMR 7296
}

Commission V, WG V/4

KEY WORDS: Photogrammetry, 3D acquisition, cultural heritage, low cost solution, web platform

\begin{abstract}
:
In recent years, advances in the fields of photogrammetry and computer vision have produced several solutions for generating 3D reconstruction starting from simple images. Even if the potentialities of the image-based 3D reconstruction approach are nowadays very well-known in terms of reliability, accuracy and flexibility, there is still a lack of low-cost, open-source and automated solutions for collecting mass of archaeological findings, specially if one consider the real (and non theoretical) contextual aspects of a digitization campaign on the field (number of objects to acquire, available time, lighting conditions, equipment transport, budget, etc...) as well as the accuracy requirements for an in-depth shape analysis and classification purpose. In this paper we present a prototype system (integrating hardware and software) for the 3D acquisition, geometric reconstruction, documentation and archiving of large collections of archaeological findings. All the aspects of our approach are based on high-end image-based modeling techniques and designed basing on an accurate analysis of the typical field conditions of an archaeological campaign, as well as on the specific requirements of archaeological finding documentation and analysis. This paper presents all the aspects integrated into the prototype:

- a hardware development of a transportable photobooth for the automated image acquisition consisting of a turntable and three DSLR controlled by a microcontroller ;

- an automatic image processing pipeline (based on Apero/Micmac) including mask generation, tie-point extraction, bundle adjustment, multi-view stereo correlation, point cloud generation, surface reconstruction ;

- a versatile (off-line/on-line) portable database for associating descriptive attributes (archaeological description) to the 3D digitizations on site ;

- a platform for data-gathering, archiving and sharing collections of 3D digitizations on the Web.

The presentation and the assessment of this prototype is based on an interdisciplinary experience carried out on the study of the Tholos in Delphi within the framework of the CNRS's Eloquentia project and the EFA's archaeological program.
\end{abstract}

\section{INTRODUCTION}

The practical aspects of a archaeological findings digitization campaign on the field such as the large number of objects to digitize, the available time, lightning conditions and transportation are today not totally taken into account by the scientific community working on 3D digitization technology design and development. In this paper, we introduce an innovative low cost, open-source and automated solution for a massive 3D acquisition of archaeological fragments on the field. We also introduce a versatile database for the on-site description of the digitized artifacts linked to an on-line platform for gathering, archiving and sharing collections of 3D digitizations on the Web.

This research campaign was conducted in 2014 as part of the program PEPS-CNRS "Eloquenzia", composed of three "Unités Mixtes de Recherche" - the "Centre Camille Jullian" (UMR 7299), the laboratory of "Modèles et simulations pour l'Architecture et le Patrimoine" (UMR 3495), and the "Laboratoire des Sciences de l'Information et des Systèmes" (UMR 7296) - with the help of the Delphi Archaeological Museum, the Ephory of Delphi and the support of the "École Française d'Athènes".

The paper is organized as follows. Section 2 presents the Tholos in Delphi. Section 3 is devoted to related work and our prototype is described is Section 4. We conclude in Section 5.

\section{CONTEXT OF THE THOLOS IN DELPHI}

2.1 The monument: status, description

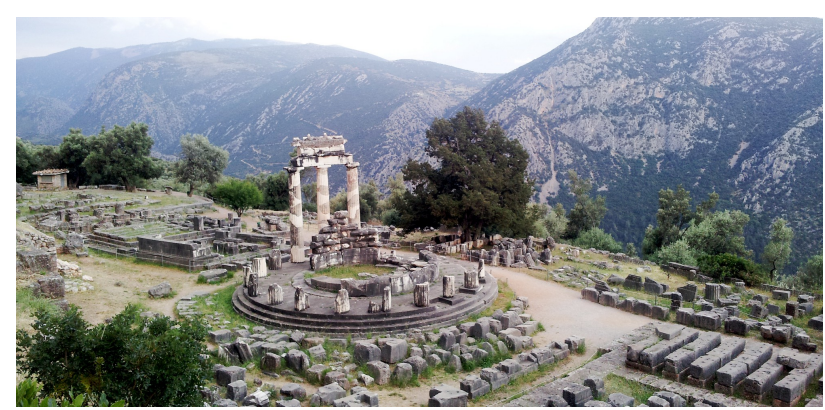

Figure 1: The Tholos in the Sanctuary of Athena Pronaia, Marmaria, Delphi, May 2014.

The archaeological site of Delphi, located in Phocis (Central Greece) and excavated by the French School of Archaeology in Athens ("École Française d'Athènes") since the end of the XIX ${ }^{\circ}$ century (The "Grande Fouille" occurred between 1892 to 1903) (Hellman, 1992a), is composed primarily of two Panhellenic sanctuaries, that of Pythian Apollo at the foot of Phaedriades on Mount Parnassus, and that of Athena Pronaia further below at the place called Marmaria. 
This landmark of Greek religion - the Omphalos or "navel of the world" - was founded at the beginning between of VIII century $\mathrm{BCE}$ and abandoned during the $\mathrm{VI}^{\circ}$ century $\mathrm{CE}$, with its period of hegemony between the $\mathrm{VI}^{\circ}$ and $\mathrm{IV}^{\circ}$ centuries $\mathrm{BCE}$ when the site was a symbol of unity in the Hellenic world.

Our current research campaign focuses on one monument in particular: the Tholos in the sanctuary of Athena Pronaia (Figure 1) . This exploratory project lays the foundation of new collaboration with the "École Française d'Athènes", which holds publication rights to the Tholos ; the publication of its sculptural decoration by Professor Philippe Jockey is forthcoming in the series of the "Fouilles de Delphes".

The monument itself has been known since 1836 but was not excavated until 1901, and published for the first time in 1925 (Charbonneaux, 1925). Despite a large number of hypotheses its function is as yet unclear (Hellman, 1992b)(Bommelaer, 1997)(Kyriakidis, 2010).

Built 370-360 BCE, the Tholos is a circular building composed of a Doric colonnade around a central naos (main room), constructed in Pentelic marble and dark limestone from Eleusis. The naos and its colonnade were both decorated with an architectural frieze of alternating triglyphs and metopes carved in high relief. According to Vitruvius, it architect was likely Theodoros of Phocaea (Vitruvius, 1995). Since Antiquity, the Tholos in Delphi has known great renown by virtue of its round plan, an original and rare feature in Greek architecture (the other Greek tholoi of the classical period are those of Athens and Epidaurus), and because of the quality of its decoration. Today it remains one of the most important monuments of the ancient Greek cultural landscape.

\subsection{Previous studies and reconstitutions}

In 1938 three of its Doric columns and entablature were reconstructed, indicative of its ongoing cultural prominence (Amandry et al., 1942). This anastylosis provides an idea of the monument proportions and of its location in the Delphic landscape, even if, paradoxically, it has only been partially reconstructed (Jockey, 2010). It should be noted, however, that a first partial reconstruction was carried out in 1902 under the aegis of the "École Française d'Athènes". The base of every column was put in place, as well as part of several courses of the naos.

Nevertheless difficulties surround our interpretation of the monument, due to the fragmentary state of the material evidence, as the result of the collapse of the building, as well as the reuse, dispersion and destruction (for lime production) of many marble blocks and slabs at the end of Antiquity. It provided this place with its modern name - Marmaria, meaning marble quarry.

This poor state of conservation is even more striking when looking at the remains of the carved metopes in high relief. The corpus comprises approximately 500 fragments of various human figures (torsos, heads, legs, arms, both male and female body parts, nude and draped), horses (which may suggest centaurs in some cases), and marble plaques. The first published study, admirably comprehensive and thorough, was carried out by Jean Marcadé in the 1970s (Marcadé, 1979a, 1979b). Using technical and stylistic criteria, he was able to identify and gather fragments pertaining to the Tholos that had been scattered and reused in later monuments, alongside those rediscovered during excavations. He was further able to reassemble some architectural elements. In this way he revealed the mythological scenes in high relief which decorated the metopes. The forty metopes of the Doric colonnade $(65 \mathrm{~cm}$ in height) depict scenes of a Gigantomachy and an Amazonomachy, whereas the forty small metopes adorning the naos (42 cm in height) depict a group of deities about whom it is difficult to analyse further at present.
Using these early studies, the first trial 3D virtual reconstruction of the monument was carried out successfully by a three-dimensional scanner in 1995 thanks to technological sponsorship (Bommelaer, 1997)(Jockey, 2012). A turntable was used to digitize some architectural fragments with a triangulation based 3D scanner (Bommelaer, 1997). Reconstruction of the metopes was limited by their fragmentary state, the very strong relief decoration and the wear of some archaeological features.

\subsection{A new research project: "Eloquenzia"}

At a time when experimentations on 3D digitization of heritage artifacts seem having a strong focus on recording and visualization issues, a new look on the cognitive issues of the digital representation seems essential.

Reasoning about shapes, their morphological, structural and semantic similarities is a fairly widespread practice in archaeology and cultural heritage. The analysis of discretised forms is also a research topic for mathematicians and computer scientists studying computer graphics and geometric modeling. To promote an interdisciplinary reflexion about this issue seems particularly interesting today, when observing geometrical features in digitized shapes could make emerge new strategies for analysing and classifying heritage artifacts by reading their morphological signature.

By combining top-down (representing the geometry from highlevel semantic descriptors, e.g. vocabulary terms) and a bottom-up (extracting semantics from low-level geometric descriptors - e.g. discrete curvatures) approaches, the CNRS PEPS Eloquentia project looks at the intersection of the two approaches in order to identify some new description models (integrating geometry \& semantics) for embedding the richness of 3D digitization into intelligible representations.

These purposes led to the current study, which combines archaeological approaches with 3D digitization and shape analysis, to test our understanding of the sculptural decoration of the Tholos. The contribution of new technologies (photogrammetry, computer vision and 3D modeling) can be seen as a logical development in the digital age to the graphic restitutions of archaeologists and architects of past centuries (Bommelaer, 1997). This close collaboration between archaeologists and computer scientists, based on a continuous and mutual experimentation/assessment process defines the basis of the experimental system presented in this paper. It will also help to better understand the architecture and carved decors of this famous monument that was in Antiquity the Tholos of Marmaria in Delphi.

Last, these new technologies could help to evidence more accurately some material or technical details, such as breaks in the marble, tools traces and final surface treatments. This study, based on both the architecture of the monument and its carved ornaments, will accompany the publication of the sculptural decoration, linked with a relational online database and $3 \mathrm{D}$ virtual anastylosis.

\section{RELATED WORK}

\subsection{Artifacts digitizations by photogrammetry}

Multi-view photogrammetry applied to dense point cloud generation becomes common today. This is explained by its flexibility and low technological requirements: a digital camera and a laptop are enough. Most software protocols (Agisoft, Bundler/PMVS) recommend between 60 and 120 images all around a simple subject to cover it entirely, but do not provide some exact information about the angles between photographs or their precise relative positions. This means that a typical manual acquisition for a statue or small artifact with one 
camera takes around 20 to 30 minutes, without the lightning installation and the high risk to forget some point of views of getting $100 \%$ of perfectly stable and sharp images.

That is the reason why recent works are focusing on new solutions for decreasing the acquisition time and for guarantying a better coverage of the artifacts by developing massive acquisition rigs with up to 100 digital reflex cameras. As a DSLR price is at least $400 €$, a rig cost is extremely high, plus they seem generally difficult to transport and adapt to different subjects. In a parallel way, the Fraunhofer CultLab3D project (cultlab, 2014), is focusing on the industrialization and automation of the entire 3D digitization process of artifacts by means of state-of-the-art scan and illumination techniques integrated into a digitization laboratory.

\subsection{Web solutions for data gathering and sharing 3D}

The range of available solutions for visualizing interactive 3D models on the web is growing today (3D PDF, WebGL, Unity, etc.) and many European projects (CARARE, 3D-ICONS, etc.) started international initiatives for making available 3D content into cultural portals (e.g. Europeana) as well as for developing metadata schemas able to enrich the 3D content with information related to the digitization process and to the understanding and interpretation of the digitized artifacts. Even in these projects are forming today the basis for the future 3D libraries of cultural content for the general public, very few works are focusing on a complete integration of the 3D digitization practice within the overall heritage artifacts documentation pipeline (including the on-site observation, classification, description, measurement, annotation, etc.). This underlines the need for versatile databases (working with and/or without internet access) able to assist the documentation process as well as to manage the archiving, the sharing and the publishing of big collections of heterogeneous data (text, photographs, 3D point clouds, 3D models, textures, etc.).

\section{PROTOTYPE DEVELOPMENT}

\subsection{Acquisition protocols}

Since 2012, the MAP laboratory, in partnership with ENSG and IGN is developing optimized photogrammetric acquisition protocols for cultural heritage artifacts documentation (archaeological fragments, paintings, sculptures, furniture, architectural elements, buildings, sites, etc.) by basing on the parametrisation of the APERO /MICMAC toolchain (PierrotDeseilligny, 2011).

This automated image-based modeling pipeline consists in tiepoints extraction (based on SIFT (Lowe, 1999) algorithm), internal and external orientations of each image by bundle adjustment (Apero), and dense image matching (Micmac). The final output is a set of dense and accurate point clouds: the correlation algorithm allows for each pixel of photograph to generate a corresponding 3D point. This means the density is relative to the focal length of the lens and its distance to the subject. A recent paper presents the metrological assessment of this automated processing pipeline by basing on the comparison with 3D laser scanning (Toschi et al., 2014).

By using the recommendations and specifications for an optimal image acquisition protocol (Martin-Beaumont et al., 2013) as starting point, we defined the requirements for a totally automated acquisition process aiming at the development of an experimental acquisition device. The initial requirements are listed below:

- For each desired points cloud take a "master" image and several closed associated images (with low ratio base to distance and important overlapping) (Pierrot-Deseilligny et al., 2011),

- Between each master image take a sufficient number of intermediary images to assure the connection during the orientation step (Figure 2),

- Avoid to move a punctual light source during the photographs acquisition,

- Use a context with enough details spread in all the space photographed as background,

- Fix as many parameters of the cameras as possible.

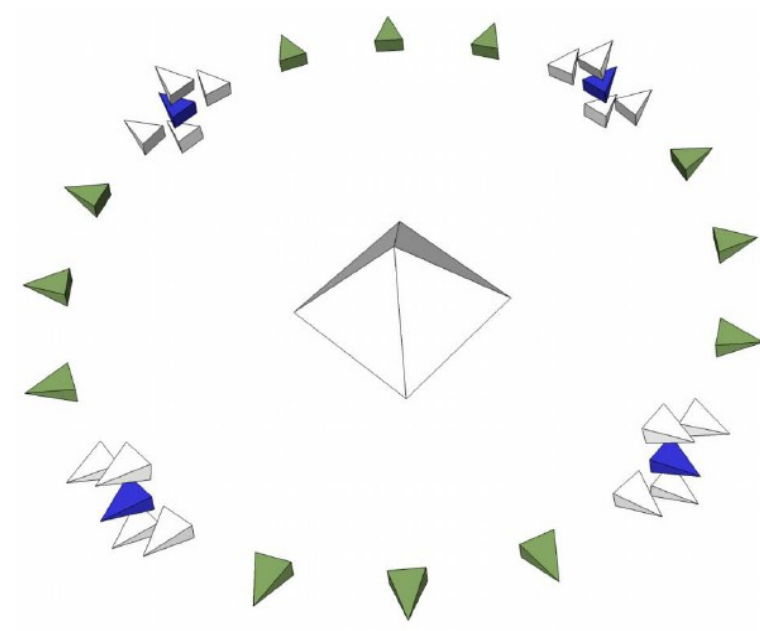

Figure 2: Spatial configuration of the acquisition of an artifact (Nicolas Martin-Beaumont, 2013)

Nicolas Martin-Beaumont (Martin-Beaumont et al., 2013) then proved that a master image needs at least 4 slave images, all around, taken all around with a angle inferior than $10^{\circ}$ for an optimal correlation.

It was then decided to move not the camera but the subject by making it spin on a turntable. Also, 3 DSLR will be used to cover the upper and lower zones of the model. This way, as many photos as wanted can be taken all around. This causes some problems regarding the recommendations: the light should not change between photographs and the context needs details spread around in the background to help the orientation part.

\subsection{Hardware development}

In accordance with museum curators, it was decided that an artifact measuring less than 60 centimeters in its largest dimension and/or having a weight less than 20 kilograms can be moved and placed on a rotating platform. Those numbers depend of course on each museum rules and policy, but it was a starting point to think about our automated acquisition solution. Also, the whole prototype has to be transportable by plane and used by one person. A large luggage measures $50 \times 80 \times 30$ centimeters and the maximum accepted weight is 32 kilograms. Finally, as a challenge, it must be the most costeffective as possible.

A turn table was salvaged from an old prototype. It could accept a weight up to 100 kilogramd, more than enough keeping in mind this project was meant to stay low cost. It was added a wheel worm drive linked to a stepper motor salvaged from an old printer to move the table. This link has two main advantages: it limits the momentum on the motor and keeps the turntable from moving when the motor is off (Figure 3). 


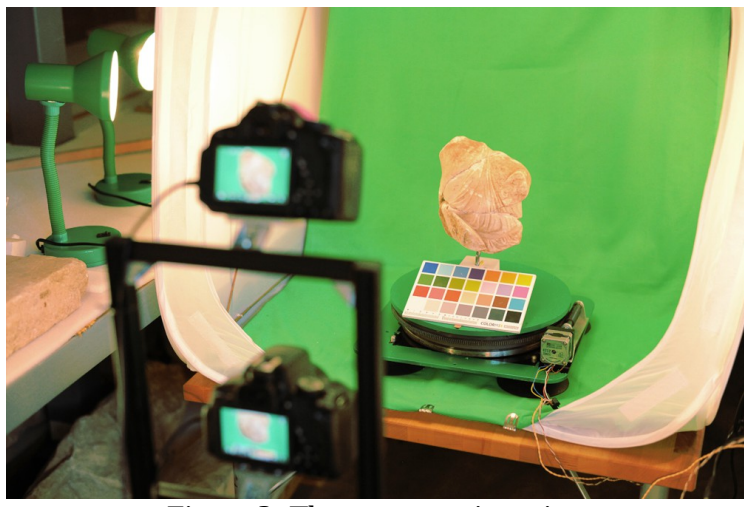

Figure 3: The prototype in action

The 3 digital cameras were already in possession of the laboratory. They are equipped with a 50mm F/1,8 lens. In the case of this first experimentation in Delphi, only the camera trigger was tethered. Each camera has still to be set manually and the memory cards tranfered one by one. The whole system is controlled by an Arduino micro-controller.

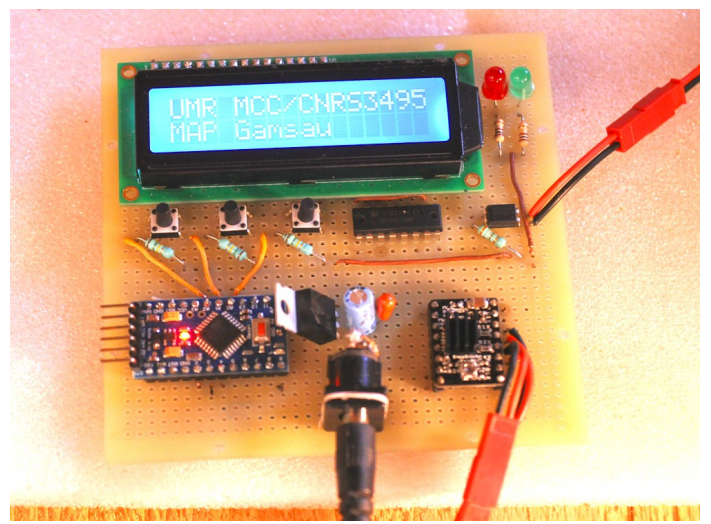

Figure 4: Dedicated electronic prototype board

A dedicated electronic prototype board (Figure 4) was developed. Its role is to distribute the power to the turntable, move it and trigged synchronously the camera. A LCD screen and three push buttons (ok/return and left/right) where added to keep the system autonomous. A simple menu allows to set the following parameters:

- Number of acquisitions in a complete rotation

- Time for each acquisition

- Number of photographs per acquisition.

As the cameras are set separately, the waiting time between two displacements has to be manually entered in the controller. Also, the option for bracketed acquisition in order to create high dynamic range images was implemented, so number of photographs per acquisition could be set up. This was yet not used.

To guarantee an optimal image orientation and a good texture quality, the lightning has to be as uniform as possible. We used a folding photo booth of $1 \mathrm{x} 1 \mathrm{x} 1$ meter size. The diffusing sides allows to create a uniform illumination. The goal of the Eloquenzia project was to study the geometric aspect of the artifacts, the textures were not the main objective. That's why we used seven cheap standard desk halogen lamps. They were provided with intensity modulators to equalize manually the ambiant light. In a last step, we mounted a green wall on the back of the photo booth. This was used after the acquisition to detach the object and create a uniform white background.

\subsection{Software and practice}

The acquisition protocol we used during the Eloquenzia mission was the following:

- While the previous object was photographed in the photo booth, the next one was referenced on our database, setting the timestamp, folder name on the camera, number of pictures and other acquisition settings

- The object was then placed on the turntable, stabilized on a weighted platform.

- The cameras were set and adjusted for the object to be seen entirely and meanwhile cover the largest possible portion of the camera's sensor.

- On each camera the image counter was reset and a new folder created. Then the exposure and aperture were set.

- The micro-controller was set if the exposure time has changed, and the number of desired photographs was set before launching the acquisition.

This whole operation lasts an average of 5 minutes for a $3 \times 75$ images acquisition.

As the counter is reset for each object, a new folder is created automatically. When emptying the memory cards, each folder name corresponds to one artifact. Then, the images for a same object for the three cameras can be moved automatically, gathered and renamed in a same folder. A simple bash script has been written for this operation. This allows an easier programming for the next steps as we already know that for each number, the image is vertically aligned with the two other camera images.

Then starts the entirely autonomous 3D generation scripts. First the images need to be clipped from the green background (Figure 6, left images). A GIMP script was written for this operation. It automatically picks a median color in 10 pixel radius zone in the upper right corner of each image and selects with a adjustable threshold all the similar colors. This selection is grown to fill the eventually missed pixels and filled with plain white. Then, as said in Section 4.1, a tie-point extraction is performed on all the images. As each image has common points only with the 5 previous and following ones, the SIFT operation is done this way. Also, the 10 last and 10 first images are matched as the acquisition is circular. Also, the images has some strong similarities with the same vertical position in the other lines. This protocol allows an optimized way to compute a maximum of tie-points in a minimum of time. The 3 cameras use the same lens, which was precisely calibrated before.
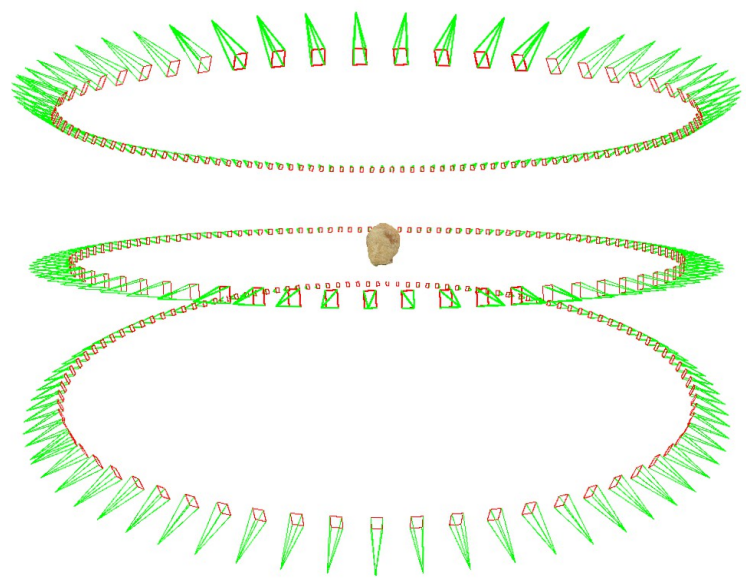

Figure 5: Orientation visualisation 
As the cameras are moved between each acquisition, it was chosen to separately compute the orientation of each object. First the central line orientation is computed, and starting from this result the upper and lower lines are oriented (Figure 5).

Once the orientation computed, a mask is automatically generated via another GIMP script. This accelerate the correlation by deleting the useless white background.

The dense correlation is made every 9 images on each line which gives 8 point cloud for each line. The master images are chosen alternatively to maximize the coverage of the object.
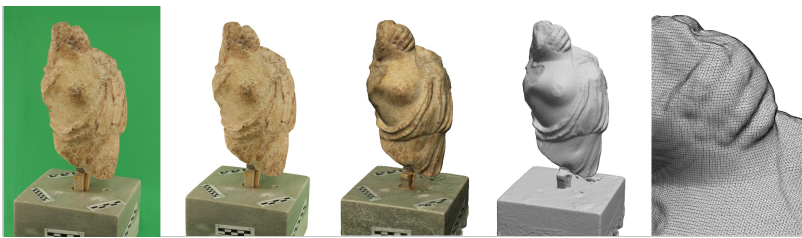

Figure 6: Different steps of the image processing:

Raw photograph; clipped image; colored 3d mesh; raw mesh; mesh detail.

The assembled result is composed of an average of five million points, with an important overlap between every separate point clouds. A mesh is then computed using the screened Poisson reconstruction software developed by (Kazdhan and Hoppe, 2013) (Figure 6, right image). The figure 6 subject is an amazon torso $\mathrm{N}^{\circ} 4312$ (Marcadé, 1979b, p161).

\subsection{Database development}

The whole data produced by the project must be stored. In order to query and manage them we implemented an SQL database containing for each archaeological object: unique id, archiving index, name, type, morphological characteristics, tools marks, photographs and a binary file containing the 3D object mesh. To be easily accessible for scientists we constructed an application framework that will permit to store, modify and share the data. The applications are embedded in a web browser to help diffusion and maintenance, and are available in-line or off-line. We plan two usages: 1) in the laboratory, assuming Internet connection, the web-browser handle a classical interface to query, modify, suppress data in a centralized database that is accessible once the user authenticated (in read-only or read-write mode). 2) while in mission, the user must edit or consult the data in an off-line mode. This is issued by exporting the database into the traveling computer. The application for the user is downloaded on the server. The user carries both data and application, thus he always has the most recent version. After the mission, the data are merged with the server ones and shared again with the community.

A special mode on the server allows an administrator to manage the database scheme and to convert from one scheme to the other one, handling the database evolution (Figure 7).

We based our developments on Javascript language, both on client (embedded in the web-browser) and server (node.js) sides. It makes easier the deployment and the maintenance of the client application and does not bother about computer or operating systems the clients work on. Each time the user opens its browser on-line the current interface is downloaded, and when on departure for a mission the latest client is included. We take advantage of the dissemination of HTML5 standard, in which 3D visualization is made possible without third-party plug-ins. WebGL is specified by the Khronos group (khronos, 2014), as OpenGL or OpenCL, it offers a direct access to the graphic hardware of the client (Joshi, 2012). A 3D canvas can be created in the HTML5 document, and
Javascript programming language is used to handle the 3D environment (in the DOM - Document Object Model - of the page). WebGL is an API that brings OpenGL ES (for "Embedded Systems") in a web browser.

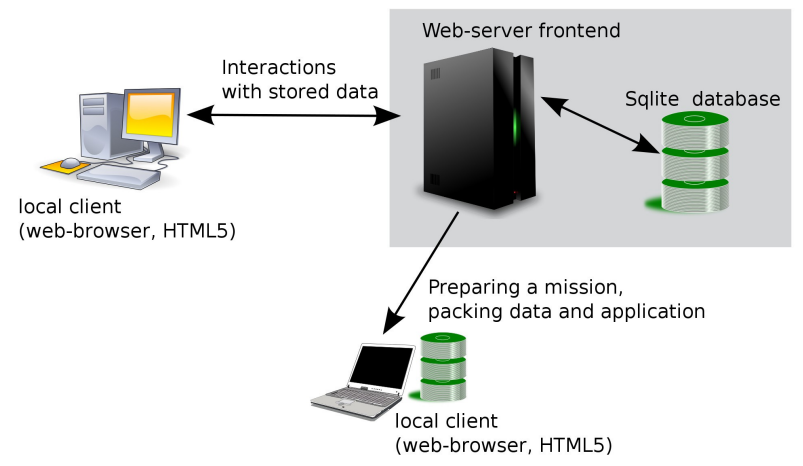

Figure 7: Offline/Online database managment

Working on the database allows the user to insert the 3D digitized mesh model he processed abroad, having a tool to view/rotate/zoom the $3 \mathrm{D}$ model. When he will return to the laboratory, the new data will be inserted as other scientific/multimedia ones.

In order to manage these new data when returning from mission, each time a value is added or modified off-line a log is create in a special table in the base, containing author ID, computer ID and field/data modified. These values are used when merging server data with the ones collected by the itinerant researchers. If a coherence problem in the fields or in the values is raised an archaeological scientist must merge manually the data (Figure 8).

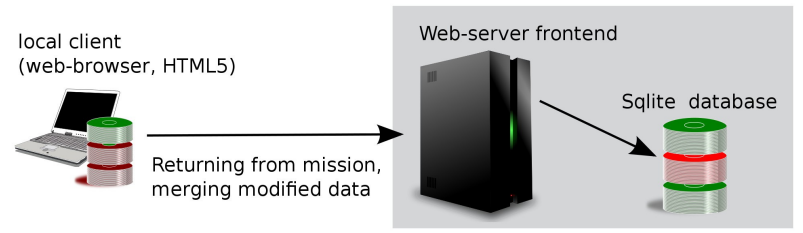

Figure 8: Database merging

\subsection{Final web publishing of the gathered data}

In order to provide the final web publication of the gathered data (including photographs, 3D point clouds, 3D models and related descriptions) we developed a specific meta-viewer, which can be easily embedded into a common webpage, able to switch the visualisation and interaction properties according to the type of content. Our meta-viewer is based on Web-Gl and Three.js and integrates a set of open source graphics libraries for managing the real time interactive visualization of all the data gathered and elaborated by the presented pipeline: 2D photographs (jpg and png format), dense and colored 3D point clouds (in ply format) by basing on (Potree, 2014), 3D textured meshes (in dae and obj formats). The meta-viewer provides the automatic configuration of the download process as well as of the interaction functions to be added to the selected content (zoom, pan, roll, ...). Finally, as it is based on standard W3C certified technologies, it is already compatible with multiple devices (PCs, laptops, tablets and smartphones). 


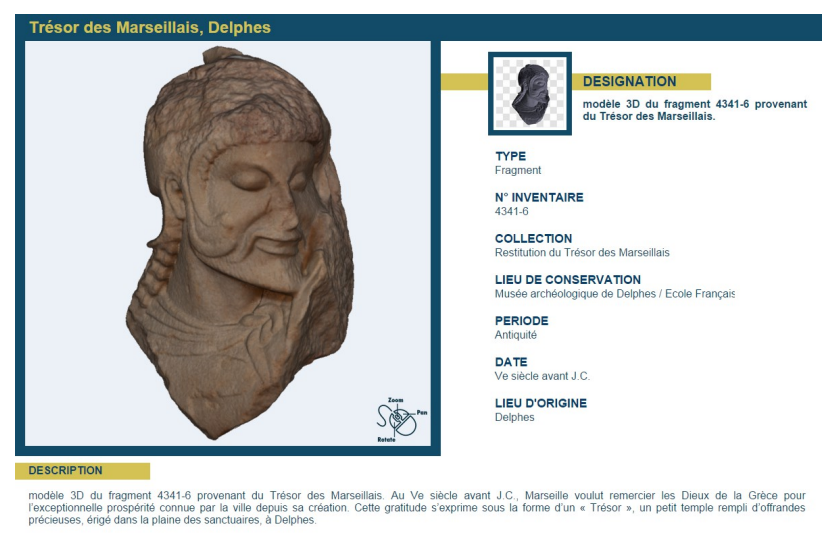

Figure 9: Screen capture of our web platform

\section{CONCLUSION}

\subsection{Assessments}

Initially, the PEPS Eloquenzia aimed at capturing a dozain significant models, that were previously carefully selected among the hundreds available. Our approach along the mission proved to be more effective than expected and we acquired in 8 days more than 150 different artifacts. This resulted in 200 gigabytes of JPEG images. In average, the raw 3D point cloud resulting from a automated generation is composed of five million points. As the images used for the point cloud generation have a important overlapping, a sampling is made before meshing. The mesh has an average one million polygons. Also, as the camera global orientation is automatically computed, there is no physical scale and reference. The $3 \mathrm{D}$ mesh has to be manually scaled thanks to scale references sticked on the object support before the acquisition.

\subsection{Perspectives}

This mission was carried out with an exploratory goal, which was outperformed. Thus, a few evolutions are still to implement:

Three cameras were not enough to properly cover the upper and lower part of an artifact. Also the illumination could greatly be improved with dedicated lights. This would result in a better color overall the model. A textured 3D object would greatly enhance the visual experience of the user. It can be combined with compression algorithms to balance quality versus file size because WebGL environment is also limited to "small" objects (65k points for example). This must be done while uploading 3D meshes to the server. It can be possible for instance to create "low-polys" representations which can be associated to normal maps to keep a visually good aspect.

The demand for automatic processes for 3D characterization, semantic recognition, reattachment propositions is increasing, involving that $3 \mathrm{D}$ data should be stored in two resolution levels: one simple for visualization, the other at the best level to implement geometry processing algorithms.

\section{ACKNOWLEDGEMENTS}

The authors wish to thank the French CNRS-PEPS program and the "École Française d'Athènes" (EFA) for their support, the Delphi Archaeological Museum and the Ephory of Delphi which provided accesses to the archaeological site and reserves.

\section{REFERENCES}

Amandry, P. and Bousquet, J., 1992. La colonne dorique de la Tholos de Marmaria. In: Bulletin de Correspondance Hellénique, LXIV-LXV, pp. 121-127.

Bommelaer, J.-Fr., 1997. Marmaria, le sanctuaire d'Athéna à Delphes. De Boccard, Paris.

Charbonneaux, J., 1925. Fouilles de Delphes, II, Topographie et architecture [4], Le sanctuaire d'Athéna Pronaia. La Tholos. De Boccard, Paris.

Hellmann, M.-Chr. et al., 1992a. La Redécouverte de Delphes. De Boccard, Paris.

Hellmann, M.-Chr., 1992b. Delphes, aux sources d'Apollon : un siècle d'archéologie française en Grèce, exposition, EFA, Athènes.

Jockey, Ph., 2010. L'anastylose et la ruine. Brèves réflexions sur la fabrication de mémoire(s) XIXe-XXe siècles. In: M. Crivello et al., Les échelles de la mémoire en Méditerranée : XIXe-XXIe siècle. Actes Sud, Arles, pp. 73-98.

Jockey, Ph., 2012. Les enjeux d'une restitution virtuelle. In: M. Garsson (dir.), Le Trésor des Marseillais : 500 av. J.-C., l'éclat de Marseille à Delphes. Somogy, Paris, pp. 102-107.

Joshi, P., Bourges-Sévenier, M., Russell, K. and Mo, Z., 2012. Graphics programming for the web. In: ACM SIGGRAPH 2012 Courses, SIGGRAPH '12. New York, NY, USA: ACM, pp. 8:1-8:75.

Kazhdan, M., and Hoppe, H., 2013. Screened poisson surface reconstruction. In: ACM Transactions on Graphics (TOG), vol. 32, no 3, p. 29.

Kyriakidis, N., 2010. Erreurs à Delphes. La tholos de Marmaria au fil des interprétations (ca 1840-1940). In: Anabases, 11, pp. 149-163.

Lowe, D.G., 1999. Object recognition from local scaleinvariant features. In: Proceedings of the International Conference on Computer Vision, Corfu, Greece (September 1999), pp. 1150-1157.

Marcadé, J., 1979a. Les sculptures décoratives de la tholos de Marmaria à Delphes : état actuel du dossier. In: Archaische und klassische griechische Plastik, Mayence, II, pp. 169-173.

Marcadé, J., 1979b. Les métopes mutilées de la Tholos de Marmaria à Delphes. In: Comptes rendus des séances de l'Académie des Inscriptions et Belles-Lettres, 123e année, n²2, 1979, pp. 151-170

Martin-Beaumont, N., Nony, N., Deshayes, B., PierrotDeseilligny, M. and De Luca, L., 2013. Photographer-friendly work-flows for image-based modeling of heritage artefacts. In: ISPRS - International Archives of the Photogrammetry, Remote Sensing and Spatial Information Sciences, XL-5/W2 (July 22, 2013): 421-424.

Pierrot-Deseilligny, M., and Clery, I., 2011. Apero, an open source bundle adjustment software for automatic calibration and orientation of a set of images. In: Proceedings of the ISPRS Commission V Symposium, Image Engineering and Vision Metrology, Trento, Italy (2-4 March 2011). 
The International Archives of the Photogrammetry, Remote Sensing and Spatial Information Sciences, Volume XL-5/W4, 2015 3D Virtual Reconstruction and Visualization of Complex Architectures, 25-27 February 2015, Avila, Spain

Toschi, I., Capra, A., De Luca, L., Beraldin, J-A., Cournoyer, L., 2014. On the evaluation of photogrammetric methods for dense 3D surface reconsctruction in a metrological context. Proceedings of ISPRS Technical Commission V Symposium, (23-25 June 2014), Riva del Garda, Italy. ISPRS Annals of the Photogrammetry, Remote Sensing and Spatial Information Sciences, Vol. II-5.

Vitruvius, 1995. De Architectura, VII, translated by B. Liou et M. Zuinghedau. Paris, Les Belles Lettres.

http://www.cultlab3d.de/

http://www.khronos.org/

http://potree.org/ 